\title{
Survey on Implementation and Analysis of Power- Constrained Contrast Enhancement Algorithm Using a Sub-band Decomposed MSR for OLED Display
}

\author{
Pallavi Ghormade ${ }^{1}$, S. K. Bhatia ${ }^{2}$ \\ ${ }^{1}$ ME Student, E\&TC Department, ICOER, Wagholi, Pune, Maharashtra, India \\ ${ }^{2}$ Assistant Professor, E\&TC Department, ICOER, Wagholi, Pune, Maharashtra, India
}

\begin{abstract}
Among all the displays available, organic light emitting diode (OLED) are the most promising displays. They do not require backlight as required to LCDs. For any picture to be human observable, the contrast should be well adjusted. Also, for any display it is more sufficient to use if it consumes less power. In this paper, various image enhancement techniques used for images are discussed. The techniques are histogram equalization, histogram modification, power constrained contrast enhancement, lightness and retinex theory, center/surround retinex, multiscale retinex, sub-band decomposed multiscale retinex, contrast enhancement using dynamic stochastic resonance, non-linear multiscale enhancement. Also, the power model for emissive displays is also discussed and the outlook of the power constrained contrast algorithm using sub-band decomposed MSR is discussed.
\end{abstract}

Keywords: Power consumption, contrast enhancement, OLED, sub-band decomposition, multi-scale retinex.

\section{Introduction}

During the $20^{\text {th }}$ century, usage of electronic displays increased extensively. The displays are classified into two types with respect to operating principle - direct view displays and projection displays. Modern displays are categorized into two - emissive and non-emissive displays. Emissive displays do not require light source while nonemissive needs light source and is capable of reflective display. The cathode ray tube (CRT), the plasma display panel (PDP), and the organic light emitting diode(OLED) are emissive displays, whereas the thin film transistor liquid crystal display(TFT-LCD) is non-emissive. Due to the advantages of less power consumption and no need of backlight, emissive displays can be used. Among which OLED is observed most useful and can replace TFT-LCD.

\subsection{OLED}

OLED device was first made in 1987 which used a novel two- layer structure with separate hole transporting and electron transporting layers such that recombination and light production occurred in the middle of organic layer. This caused in a reduction in operating voltage and developments in efficiency. These advantages led to more production of OLED.

A typical OLED is composed of a layer of organic materials located between two electrodes, anode and cathode, all placed on a substrate. The organic molecules are electrically conductive. Many modern OLED have a simple bilayer structure consisting of conductive layer and an emissive layer. Overall, OLED is a flat light emitting equipment, made by placing a series of organic thin films among two conductors. When electrical current is applied, a bright light is released. OLEDs can be used to make displays and lighting. Because OLEDs produce light they do not require a backlight and so are thinner and more proficient than LCD.

\section{OLED structure}

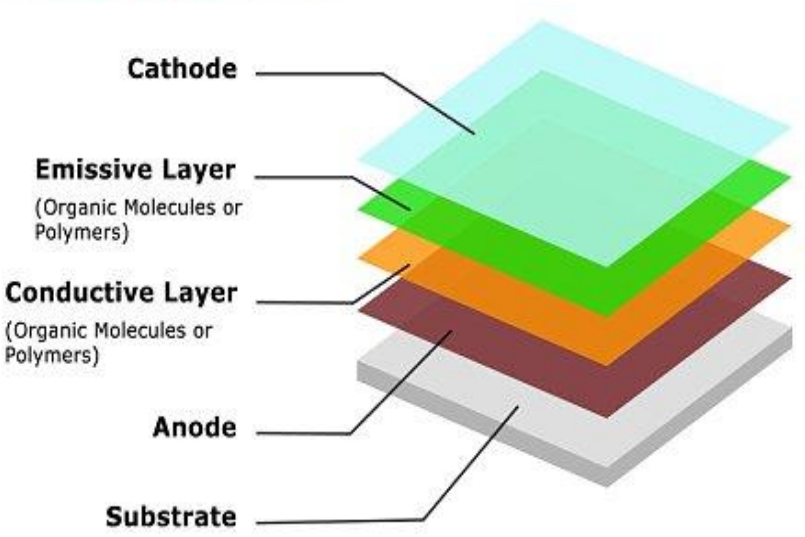

Figure 1: OLED structure

There are several types of OLEDs - passive matrix OLED, active matrix OLED, transparent OLED, top-emitting OLED, foldable OLED, white OLED. Each type has different uses.

\section{Related Work}

This section provides a study of general techniques and more particular approaches for contrast enhancement and low power intake in OLEDs. Power saving is an main issue in multimedia devices because a large portion of the power is used up by the display panels of the devices. Contrast enhancement is essential for better picturing of dark images to increase visual view and to enable exact explanation. 


\section{International Journal of Science and Research (IJSR) \\ ISSN (Online): 2319-7064 \\ Index Copernicus Value (2013): 6.14 | Impact Factor (2014): 5.611}

Several images have very low dynamic range of intensity values because of inadequate illumination and therefore needs to be treated before displaying it. Many techniques have concentrating on enhancement of gray-level images like histogram equalization, gamma correction, high pass filtering, low pass filtering etc.

\subsection{Histogram equalization}

Histogram equalization (HE) is one of the mostly implemented to improve low-contrast images, that makes the histogram of light intensities of image as even as possible. In this method, areas of lower limited contrast are allowed to gain a higher contrast which is done by effectively increasing out most frequently used intensity values. But it has the disadvantage of contrast over widening, mood modifications or contour objects in the output image. So to overcome these drawbacks histogram modification (HM) technique was proposed.

\subsubsection{Histogram Modification}

$\mathrm{HM}$ is the method that employs the histogram information in an input image to be obtaining the transformation function. HE can be observed as the superior case of the HM. In modified HM, the input histogram before the HE procedure to lessen slopes in the transformation function, in its place of the direct control of the histogram.

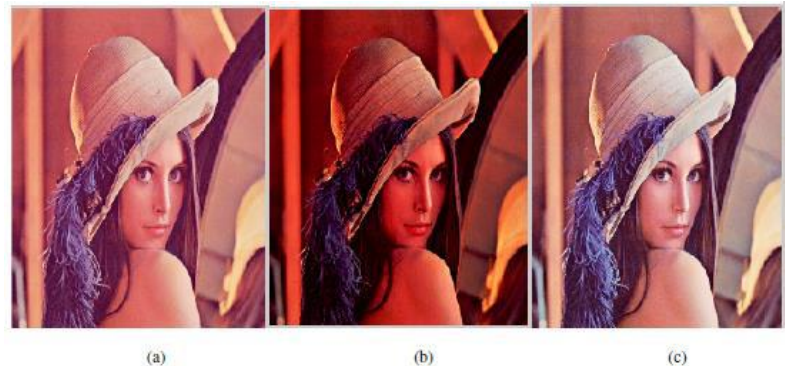

Figure 1: (a) original image (b) Enhanced Image by using HE (c) Enhanced image using HM

\subsection{Power -Constrained Contrast Enhancement}

C.Lee et al. (2012) recommended power constrained contrast algorithm for OLED displays where they demonstrated power consumption in OLED displays. They experimentally presented power to display single color pixel with respect to red, blue and green pixels strength. The pixel intensities are well-organized to save power. The same was done with emissive displays first. A power model for emissive displays was designed which was applicable for OLEDs also.

\subsection{Lightness and Retinex Theory}

Land and McCann (1971) stated lightness and retinex theory. It stated that sensations of color have a strong relation with reflectance; the quantity of light reaching the eye is a product of reflectance and illumination. The theory of retinex assumes that there are three independent cone systems with a set of receptors peaking in the long, medium and short wavelength regions of visible spectrums. It is named retinex as it employs much of the structure and function of retina necessary for producing image.
The color sensation for any area is determined by the three lightness arrived independently by three retinexes. The function of retinex theory is to tell how the eye can determine reflectance in a field in which the illumination is unknowable and the reflectance is unknown.

\subsection{Center/Surround retinex}

Jobson and Rahman (1997) executed retinex theory and came across the problems which they determined in surround retinex theory. Their form consists of placement of log function, canonical gain for concluding action for the final retinex output signals etc. It remains to simplify the retinex giving out to handle and improve the final treatment of retinex outputs. Complete performance of the retinex chains dynamic range compression, color dependability, and lightness and color interpretation. The trade-off between dynamic range compression and color interpretation ruled by surround space constant gets a multiscale method for retinex processing.

\subsection{Multiscale retinex (MSR)}

Jobson and Woodell (1997) proposed MSR, included three scales- small, intermediate and large. It was found to produce dynamic range compression, color regularity, and tonal rendition. The results were useful for human visual perception. They defined a color restoration arrangement that produced good color interpretation even for severe gray destructions.

\subsubsection{Sub-band Decomposed MSR (SD-MSR)}

Jang, Choi et. al (2008) tested sub-band decomposed MSR comparing it with standard MSR. Retinex outputs in the standard MSR algorithm have coinciding spectral ranges. So it is not capable to apply gains to retinex outputs according to their spectral characteristics. To solve this problem, they evaluated SD-MSR in which revised retinex output were disintegrated into non overlapping spectral bands.

In the evaluated SD-MSR, facts of highpoints and dark areas were explored. They also applied space varying gain which upgraded the image contrast.

\subsection{Contrast Enhancement using Dynamic Stochastic Resonance}

Jha and Biswas (2012) recommended contrast enhancement using stochastic resonance. Stochastic resonance (SR) is a happening in which noise can be used to advance the system performance. The distinctive feature of this method is that it regulates the strength values according to the bistable doublewell system parameters and exploits internal noise because of absence of illumination of a low-contrast image. The iterative process simplifies transition of the image from noisy (lowcontrast) state to good contrast state, in similar to the interwell transition of a particle in a bistable system. It is an automatic process that not only adjusts background illumination, but also increases the contrast and colorfulness while conserving perceptual quality. 


\section{International Journal of Science and Research (IJSR) \\ ISSN (Online): 2319-7064}

Index Copernicus Value (2013): 6.14 | Impact Factor (2014): 5.611

\subsection{Non-Linear multiscale image enhancement}

Nercessian and Panetta (2013) proposed an algorithm which is capable of adjusting brightness level of image straight and used a non-linearly representing to contrast coefficients at each scale. This mapping was capable of providing both active range compression and contrast enhancement. In combination with the brightness control, this method is able to achieve limited and total enhancement within a direct enhancement framework.

\subsection{Power model for OLED displays}

Energy consumption is an key design for displays. so there is a need of OLED display power models for use at different layers and different phases of system design. Power model was developed by Dong and Choi (2009)for OLED displays.

\subsubsection{Pixel level power model}

This model assumes that the power consumption of OLED modules based on RGB specification of each pixel. Power consumed by red pixels, green pixels and blue pixels are calculated individually and total power is calculated.

\subsubsection{Image Level Power Model}

Image level power model are important for a system to access the display power cost when the pixel information is known. So for this model, approximation of power based on a small subset of signals or sampling is done. It also needs RGB information for all pixels.

\subsubsection{Code-level Power Model}

Code level model is more useful than the sampling based on the code specification of the display content because they do not need access to frame buffer. Therefore it can be readily applied as an application.

\section{Proposed System}

According to the earlier work, it is studied that using power modeling it can power consumption of OLED can be controlled. Using sub-band decomposition, the retinex outputs are divided into non-overlapping spectral bands. Combining it with multiscale retinex, contrast enhancement can be achieved. Also dynamic range compression using an adaptive weighting approach power for input image is achieved.

\section{Conclusions}

The above existing techniques are not able to complete contrast enhancement with less power consumption. So a method of contrast and power enhancement in a simultaneous process is introduced using power constrained contrast enhancement for OLED displays as they can replace LCDs further.

\section{Acknowledgement}

With all respect and gratitude, I would like to thanks all people who have helped me directly or indirectly for the completion of this Project seminar.

I express heartily gratitude towards Prof. S. K. Bhatia for guiding me to understand the work conceptually and for her constant encouragement to complete this work and also for providing all necessary information.

I also express my thanks to Prof. P. R. Badadapure, Head of the department of electronics and telecommunication engineering for providing all necessary information.

With deep sense of gratitude I thank to our Principal Dr. S.V. Admane and management of Imperial College of engineering for providing all necessary facilities and their constant encouragement and support.

\section{References}

[1] K. Suzuki, "Past and future technologies of information displays," in Proc. IEEE IEDM, Dec. 2005, pp. 16-21.

[2] C. Lee, C. Lee, Y.-Y. Lee and C.-S. Kim, "Powerconstrained contrast enhancement for emissive displays based on histogram equalization," IEEE Trans. Image Process., vol. 21, no. 1, pp. 80-93, Jan. 2012.

[3] C. Lee, C. Lee, and C.-S. Kim, "Power-constrained contrast enhancement for OLED displays based on histogram equalization,” in Proc. IEEE ICIP, Sep. 2010, pp. 1689-1692.

[4] E. H. Land and J. McCann, "Lightness and retinex theory," J. Opt. Soci. Amer., vol. 61, no. 1, pp. 1-11, Jan. 1971.

[5] D. J. Jobson, Z.-U. Rahman, and G. A. Woodell, "Properties and performance of a center/surround retinex," IEEE Trans. Image Process., vol. 6, no. 3, pp. 451-462, Mar. 1997.

[6] D. J. Jobson, Z.-U. Rahman, and G. A. Woodell, “A multiscale retinex for bridging the gap between color images and the human observation of scenes," IEEE Trans. Image Process., vol. 6, no. 7, pp. 965-976, Jul. 1997..

[7] J. H. Jang, B. Choi, S. D. Kim, and J. B. Ra, "Sub-band decomposed multiscale retinex with space varying gain," in Proc. IEEE ICIP, Oct. 2008, pp. 3168-3171.

[8] S. C. Nercessian, K. A. Panetta, and S. S. Agaian, "Nonlinear direct multi-scale image enhancement based on the luminance and contrast masking characteristics of the human visual system," IEEE Trans. Image Process., vol. 22, no. 9, pp. 3549-3561, Sep. 2013.

[9] R. Chouhan, R. K. Jha, and P. K. Biswas, "Enhancement of dark and low-contrast images using dynamic stochastic resonance,” IET Image Process., vol. 7, no. 2, pp. 174-184, Sep. 2013.

[10]M. Dong, Y.-S. K. Choi, and L. Zhong, "Power modeling of graphical user interfaces on OLED displays," in Proc. ACM/IEEE DAC, Jul. 2009, pp. 652-657 


\section{Author Profile}

Pallavi T Ghormade has Completed BE from RTM Nagpur

University. Currently pursuing ME in signal processing from ICOER Pune.

Prof. S. K. Bhatia is Assistant Professor at ICOER Pune. 